\title{
Effect of different factors on the electricity consumption and electricity usage intensity (EUI) of residential buildings in Pakistan
}

\section{Efecto de diferentes factores sobre el consumo de electricidad y la intensidad de uso de la electricidad (EUI) en los edificios residenciales en Pakistán}

Khuram Pervez Amber (Main and Corresponding Author)

Department of Mechanical Engineering, Mirpur University of Science and Technology (MUST)

Mirpur-10250 (AJK) (Pakistan)

khruam.parvez@must.edu.pk

\section{Muhammad Umer Saeed}

Department of Mechanical Engineering, Mirpur University of Science and Technology (MUST)

Mirpur-10250 (AJK) (Pakistan)

sahilabbasi39@gmail.com

\section{Muhammad Waqar Aslam}

Department of Computer Systems Engineering, Mirpur University of Science and Technology (MUST)

Mirpur-10250 (AJK) (Pakistan)

waqar.cse@must.edu.pk

\section{Imdad Hussain}

Department of Mechanical Engineering, Mirpur University of Science and Technology (MUST)

Mirpur-10250 (AJK) (Pakistan)

imdad.hussain@must.edu.pk

\section{Muhammad Sajid Khan}

Department of Mechanical Engineering, Mirpur University of Science and Technology (MUST)

Mirpur-10250 (AJK) (Pakistan)

sajid.me@must.edu.pk

Manuscript Code: 1089

Date of Acceptance/Reception: 17.11.2018/20.03.2018

DOI: 10.7764/RDLC.17.3.473

\begin{abstract}
Buildings consume one-third of final energy consumption in the world and are graded as the largest sources of carbon dioxide ( $\left.\mathrm{CO}_{2}\right)$ emissions. Buildings energy consumption is driven by numerous factors that have direct or indirect effects on it. Identification of such factors could help building designers and architectures in introducing energy efficient designs of various types of buildings. Such studies are indispensable for countries such as Pakistan where a very limited research has been published in this area. This study attempts to fill this knowledge gap by investigating the effect of numerous factors on the residential electricity consumption and further, aims to establish an electricity usage intensity (EUI) value for the residential sector of Pakistan. Data were collected from 523 dwellings through site surveys and interviews with residents. Effect of 48 different variables, (five socioeconomic variables, 14 dwelling variables, 22 variables from electric appliances details, five demographic variables and two variables from renewable energy sources) was investigated on the residential buildings annual electricity consumption using SPSS software. It was found that 17 out of 48 variables have significant linear relationship with annual electricity consumption. Further, through a multivariate analysis, it was found that annual electricity usage intensity (EUI) for Pakistan's residential buildings is $24 \mathrm{kWh} / \mathrm{m}^{2}$. Finally, this EUI value is compared with the same of two major South Asian countries. It is observed that mean EUI of Pakistan is almost similar to that of India, i.e. $24 \mathrm{kWh} / \mathrm{m}^{2}$ whereas Bangladesh has a higher value of mean EUI, i.e. $32 \mathrm{kWh} / \mathrm{m}^{2}$.
\end{abstract}

Keywords: Electricity consumption, EUI, buildings, Pakistan.

\section{Resumen}

Los edificios consumen un tercio del consumo final de energía en el mundo y están clasificados como las mayores fuentes de emisiones de dióxido de carbono (CO2). El consumo de energía en los edificios se debe a numerosos factores que tienen efectos directos o indirectos. La identificación de tales factores podría ayudar a los diseñadores y arquitecturas de edificios a introducir diseños de eficiencia energética de varios tipos de edificios. Tales estudios son indispensables para países como Pakistán, donde se ha publicado una investigación muy limitada en esta área. Este estudio intenta llenar este vacío de conocimiento al investigar el efecto de numerosos factores en el consumo de electricidad residencial y, además, tiene como objetivo establecer un valor de intensidad de uso de electricidad (EUI) para el sector residencial de Pakistán. Se recolectaron datos de 523 viviendas a través de encuestas de sitio y entrevistas con residentes. El efecto de 48 variables diferentes (cinco variables socioeconómicas, 14 variables de vivienda, 22 variables de detalles de electrodomésticos, cinco variables demográficas y dos de fuentes de energía renovables) se investigó sobre el consumo anual de electricidad de los edificios residenciales utilizando el software SPSS. Se encontró que 17 de las 48 variables tienen una relación lineal significativa 
Electricity is a major energy source for every nation and plays a vital role towards the economic development (Amber, Aslam \& Hussain, 2015). In the era of globalization, rapidly expanding reliance of nations on energy show that it will be one of the most concerning issues of mankind in the following century (Stern, 1993). Buildings consume one-third of final energy consumption of the world and are graded as the largest sources of carbon dioxide $\left(\mathrm{CO}_{2}\right)$ emissions. Building sector is one of the major energy consuming sector having a share of $40 \%$ worldwide (Domínguez, Alonso, Morán, Prada, \& Fuertes, 2015). Building sector of the United Kingdom, Europe and USA consumes about $43,40,40 \%$, respectively (United Nations, 2013; Ghedamsi, Settou, Gouareh, Khamouli, Saifi, \& Recioui, 2015). Building sector can be further be categorized as, residential and non-residential buildings. The residential sector is a major user of energy. Energy consumption trend in the residential sector of the World and of Asia is shown in Figure 1 for the period 1990-2016.

It is apparent from Figure 1 that residential sector electricity consumption has increased linearly (4\%/year) over the period of 1990-2016 (Enerdata, 2018). Energy usage in residential sector increases with an increase in population, improvement in standard of living, urbanization and migration to large cities (Ghedamsi, Settou, Gouareh, Khamouli, Saifi, Recioui, \& Dokkar, 2016). Figure 1 also demonstrates that residential energy consumption in the Asian region of the world is following the trend of world's total energy consumption. This linear increase in the residential energy consumption shows that the Asian countries would require sufficient energy resources to meet their future energy demands. Buildings sector of Pakistan consumes nearly 55\% of country's total electricity annual basis (HDIP, 2015). Sector wise electricity consumption of Pakistan (Nasir, Tariq \& Arif, 2008) is presented in Figure 2. It is apparent that the building sector is the highest electricity consuming sector in Pakistan whereas the industrial sector is the second highest electricity consuming sector with an annual electricity consumption share of $28.4 \%$. Pakistan's per capita electricity consumption is $438.26 \mathrm{kWh}$ which is $3.4,7,17$ and $87 \%$ of USA, Europe, China and India's per capita electricity consumption, respectively (Nation Master, 2018).

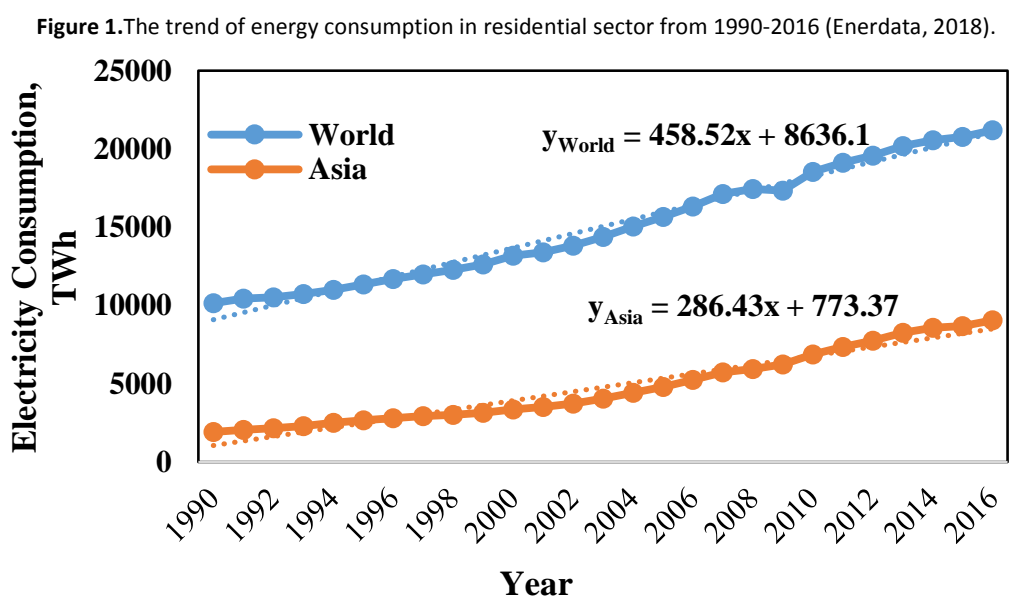

The trend of electricity consumption in residential sector of Pakistan from year 2009-10 to year 2014-15 is shown in Figure 3. It is apparent that the electricity usage in residential sector of Pakistan remained fairly constant from 2009-10 to 2012-13. After 2012-13, the electricity consumption has started increasing (Government of Pakistan, Ministry of Finance, 2006). Punjab province has the highest share with Sindh, KPK and Baluchistan provinces at the $2^{\text {nd }}, 3^{\text {rd }}$ and $4^{\text {th }}$ place. From the current trend it is expected that the electricity consumption will be increased $39 \%$ by 2025 compared to 2015.

Different countries have different cultures and climate and therefore, it is very important to individually investigate the effect of different types of factors on buildings energy consumption. Table 1 explains the work of the researchers and their use of different statistical techniques to determine the variables having significant impact on the energy consumption in buildings sector. 
Figure 2. Sector wise electricity consumption of Pakistan (HDIP, 2015).

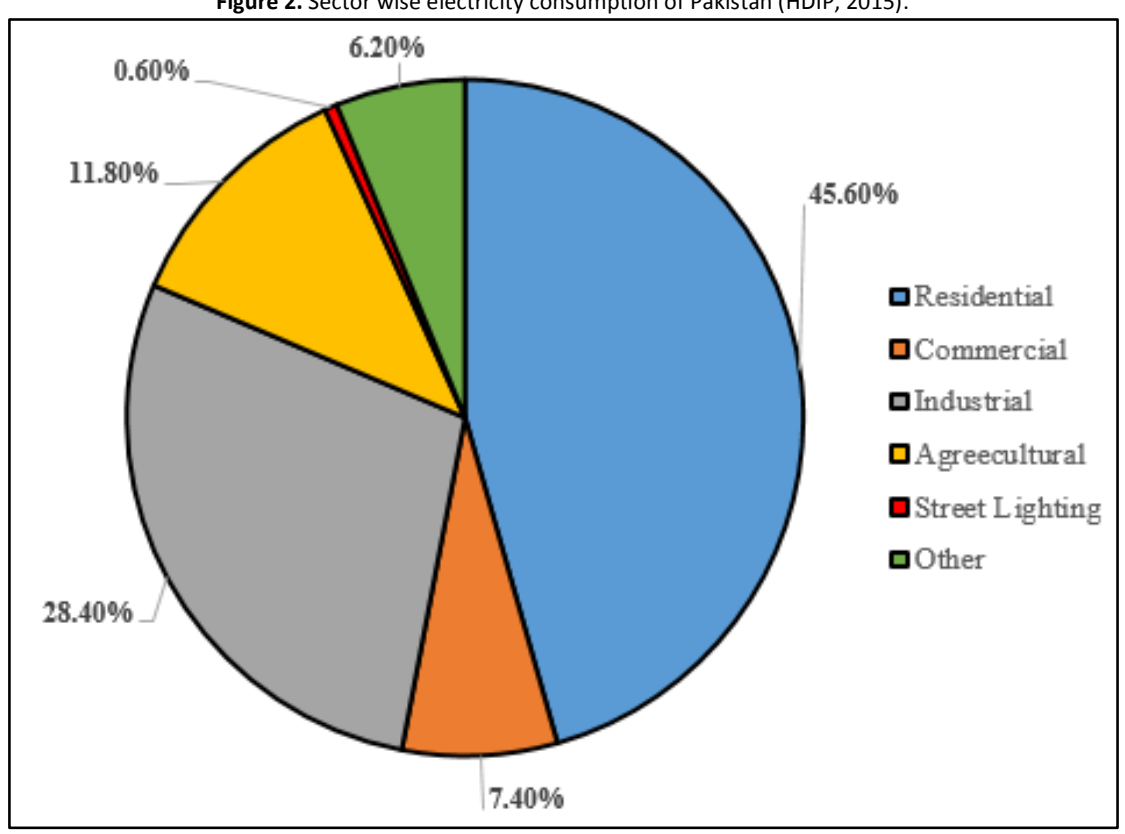

Figure 3. Electricity usage trend in residential sector of different provinces of Pakistan (HDIP, 2015).

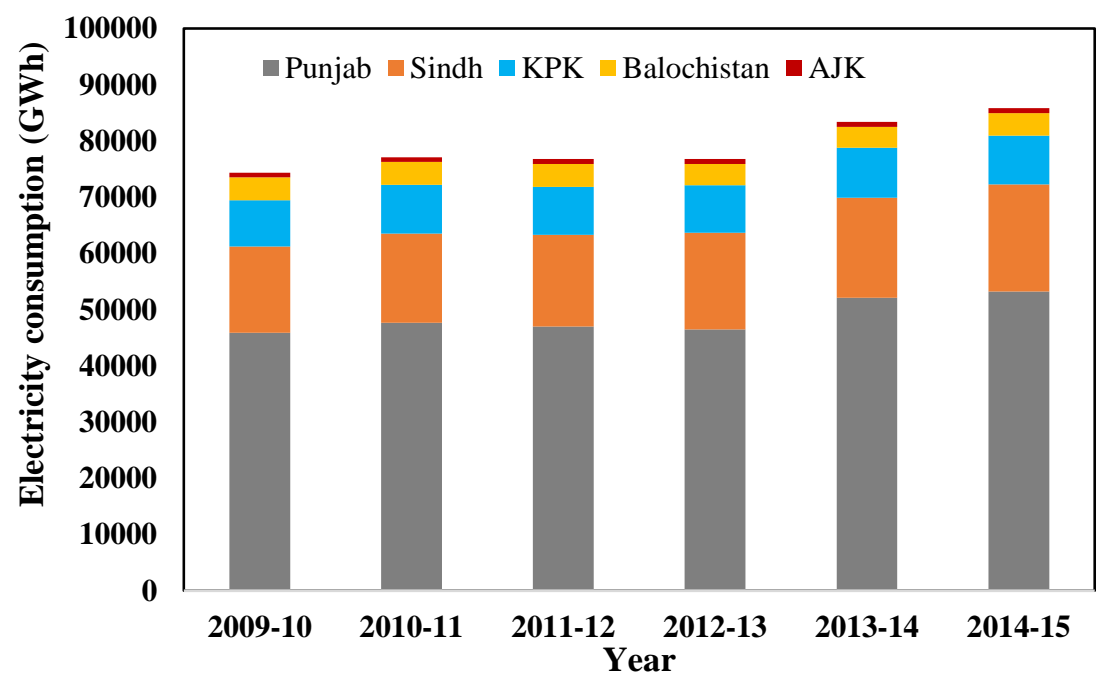

On the basis of above literature review the variables effecting the electricity consumption can be divided into four major categories, i.e. dwelling variables, socio-economic variables, electric appliances variables and demographic variables. The variables having significant impact on the annual residential electricity consumption are categorized below.

\section{Dwelling variables}

It is observed that the use of double pan window and south facing orientation can reduce the electricity consumption (Kavousian, Rajagopal, \& Fischer, 2013). The size of home (Baker \& Rylatt, 2008; William \& Gomez, 2016; Huebner, Shipworth, Hamilton, Chalabi \& Oreszczyn, 2016), number of bedrooms (Baker \& Rylatt, 2008; Jones, Fuertes, \& Lomas, 2015), building orientation (Abanda \& Bayers, 2016), age of building (Jones, Fuertes, \& Lomas, 2015) and type of home (Durkman \& Jackson, 2008) have also significant positive impact on the annual electricity consumption of the home. Also the single family detached homes consume more than $75 \%$ of the residential electricity. 


\begin{tabular}{|c|c|c|c|}
\hline Author (s) & Country & $\begin{array}{l}\text { Sample } \\
\text { size } \\
\text { (No.) }\end{array}$ & $\begin{array}{c}\text { Investigated } \\
\text { variable categories }\end{array}$ \\
\hline $\begin{array}{l}\text { Kavousian, } \\
\text { Rajagopal, } \\
\text { \& Fischer, } \\
2013\end{array}$ & USA & 1,628 & $\begin{array}{c}\text { Weather and } \\
\text { location, Dwelling } \\
\text { variables, } \\
\text { Appliances } \\
\text { variables, } \\
\text { occupancy and } \\
\text { occupants, } \\
\text { behavioral factors }\end{array}$ \\
\hline $\begin{array}{l}\text { Baker \& } \\
\text { Rylatt, } 2008\end{array}$ & UK & 148 & $\begin{array}{l}\text { Dwelling Appliance } \\
\text { Socio-demographic }\end{array}$ \\
\hline $\begin{array}{l}\text { Tso \& Yao, } \\
2003\end{array}$ & $\begin{array}{l}\text { Hong } \\
\text { Kong }\end{array}$ & 1,500 & $\begin{array}{c}\text { Dwelling, } \\
\text { Appliances, } \\
\text { demographic, } \\
\text {,economic }\end{array}$ \\
\hline $\begin{array}{l}\text { William \& } \\
\text { Gomez, } \\
2016\end{array}$ & USA & 426,305 & $\begin{array}{c}\text { Dwelling, Fuel type } \\
\text { (electricity, natural } \\
\text { gas, both) }\end{array}$ \\
\hline $\begin{array}{l}\text { Abanda \& } \\
\text { Bayers, } 2016\end{array}$ & & & Building orientati \\
\hline $\begin{array}{l}\text { Sanquist, } \\
\text { Orr, Shui, \& } \\
\text { Bittner, } \\
2012\end{array}$ & USA & 2,165 & $\begin{array}{l}\text { Lifestyle, house } \\
\text { hold income, } \\
\text { weather and } \\
\text { behavioral factors } \\
\text { connected with } \\
\text { utilization of } \\
\text { electric appliances }\end{array}$ \\
\hline $\begin{array}{l}\text { Jones, } \\
\text { Fuertes, \& } \\
\text { Lomas, } 2015\end{array}$ & & $\begin{array}{l}\text { Compar } \\
\text { ative } \\
\text { study }\end{array}$ & $\begin{array}{c}\text { socio-economic, } \\
\text { dwelling and } \\
\text { appliances variable }\end{array}$ \\
\hline
\end{tabular}

45 degrees from north as worst and dryer, washing machine, range hood, electric heater, Increased household income, disposable income, number

material, No. of

windows and

windows

orientation, HVAC

appliances, Floor area

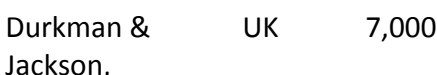
2008
Socio-economic variables, Dwelling variables
No. of bedrooms, No. of part time servants

Size of the home, Air conditioners, washing machine dryers, cooling fans, ventilation fans, number of occupants, income Size of home, Fuel type 180 degrees from north (south facing) best orientation.

$40 \%$ difference in electricity consumption due to five lifestyle factors, social and behavioral examples connected with use of PC, TV, cooling and Clothing.

Dwelling age, no. of rooms, no. of bedrooms, total floor area, level of detachment, Ownership of PC, TV, refrigerator, dishwasher, tumble of occupants and age of occupants. Size of home, type of home, no. of children's, Presence of disable person, employment status, no. of bulbs, freezers, TV, tumble dryer, dishwasher, longer lightening hours

\section{Floor area}

Cluster analysis, Multiple Regression Multiple Regression

Linear Regression, Multiple Regression, Multivariate adaptive regression Emerging BIM (building information modelling)

Factor analysis, Multiple regression

Comparative study

Multiple Regression

Ordinary least square (OLS), Support Vector Machines (SVM), Engineering Modelling (EM)

Income, type of home, location and house hold composition
Local area resource analysis (LARA), Cluster Analysis

\section{Socio-Economic Variables}

The resident's income have a significant impact on the electricity consumption (Amber, Aslam \& Hussain, 2015; Tso \& Yao, 2003; Durkman \& Jackson, 2008). For an annual increase of US $\$ 27000$ in the income of the resident, the $350 \mathrm{kWh}$ increase in the electricity consumption is seen in Japan (Genjo, Tanabe, Matsumoto, Hasegawa, \& Yoshino, 2005). Also the social lifestyle and comfort level of the residents have a strong impact on annual electricity consumption (Sanquist, Orr, Shui, \& Bittner, 2012). 


\section{Appliances variables}

The relationship of appliances and energy consumption is discussed in many studies (Kavousian, Rajagopal, \& Fischer, 2013; Tso \& Yao, 2003; Jones, Fuertes, \& Lomas, 2015; IEA, 2008; Huebner, Shipworth, Hamilton, Chalabi \& Oreszczyn, 2016). The entertainment devices, refrigerators, high utilization appliances (Kavousian, Rajagopal, \& Fischer, 2013; Jones, Fuertes, \& Lomas, 2015), air conditioners, cooling ventilation fans, washing machine dryers, lightening and cooking appliances (Tso \& Yao, 2003), water heating appliances (IEA, 2008), freezers, tumble dryers, washing machines (Huebner, Shipworth, Hamilton, Chalabi \& Oreszczyn, 2016) and building maintenance appliances (Jones, Fuertes, \& Lomas, 2015) have significant positive effect on the annual residential electricity consumption. The use of efficient lights and appliances can result in the reduction of annual electricity consumption (Kavousian, Rajagopal, \& Fischer, 2013).

\section{Demographic Variables}

It is observed that the number of residents (Kavousian, Rajagopal, \& Fischer, 2013; Tso \& Yao, 2003) and the age of the residents (Kavousian, Rajagopal, \& Fischer, 2013; Baker \& Rylatt, 2008) have significant impact on the annual residential electricity consumption. Homes with two person consume $19 \%$ more electricity per week then the home with one occupant (Leahy \& Lyons, 2010) also in India the two-family member home consumes $23 \%$ less electricity then the fivefamily member home (Tiwari, 2000).

\section{Methodology}

The data used for this study were collected from the residential sector by surveys and interviews. Three techniques for data collection were used. First of all a comprehensive questionnaire was developed in Microsoft Excel 2013 on the basis of literature review and primary surveys. Primary surveys were done do identify more independent variables which may affect the annual electricity consumption. The questionnaire was then sent to the employees of Mirpur University of science and technology via e-mail with the help of Network and Telecommunication Centre (NTC). Unfortunately out of 600 only eight employees responded. Therefore, printed questionnaires were used for door to door data collection survey. Data related to different social, dwelling and appliances factors and annual electricity consumption were collected whereas electricity consumption data were collected in the form of past one year electricity bills. The data from 600 homes were collected.

In response, 77 questionnaires (13\%) received were found to be incomplete and with some vague data. Such data was excluded on the basis of the following reasons:

- Fix electricity consumption throughout the year, i.e. no variability in electricity consumption; and,

- Questionnaires with missing details.

Out of 600 data questionnaires 25 (4\%) were excluded due to fix electricity consumption throughout the year. 32 (5\%) data questionnaires were excluded due to missing details and $20(3 \%)$ due to missing floor area details respectively. 523 (87\%) respondents returned completed questionnaire. In next sections, different datasets received as part of questionnaires are explained.

\section{Independent variables}

Variables used for data collection and regression analysis are discussed in this section. Tables 2, 3, 4 and 5 show the selected variables from dwelling, demographic, socio-economic and appliances categories respectively, their data source, reason of selection and their frequencies. 


\begin{tabular}{|c|c|c|c|c|}
\hline Variable & $\begin{array}{c}\text { Variable } \\
\text { description }\end{array}$ & Reference & $\begin{array}{c}\text { Data } \\
\text { source }\end{array}$ & $\begin{array}{c}\text { Categories } \\
\mathrm{N} \text { (Frequency), Mean }(M) \text {, Standard Deviation (SD) }\end{array}$ \\
\hline$x_{1}$ & City & $\begin{array}{l}\text { Durkman \& Jackson } \\
\text { (2008) }\end{array}$ & Site Visit & $\begin{array}{c}\text { Mirpur (N: 356) } \\
\text { Muzaffarabad (N: 167) }\end{array}$ \\
\hline$x_{2}$ & Province & & & AJK (N: 523) \\
\hline$x_{3}$ & $\begin{array}{l}\text { Type of } \\
\text { home }\end{array}$ & $\begin{array}{c}\text { Jones, Fuertes, \& } \\
\text { Lomas, 2015; EIA, } \\
\text { 2015; Huebner, } \\
\text { Shipworth, Hamilton, } \\
\text { Chalabi \& Oreszczyn, } \\
2016\end{array}$ & Site Visit & $\begin{array}{c}\text { Single storey }(N=320) \text { Multi-storey }(N=191) \text { Flat }(N= \\
\text { 9) Residential Plaza }(N=3)\end{array}$ \\
\hline$x_{4}$ & $\begin{array}{l}\text { No. of } \\
\text { stories }\end{array}$ & $\begin{array}{l}\text { Huebner, Shipworth, } \\
\text { Hamilton, Chalabi \& } \\
\text { Oreszczyn, } 2016\end{array}$ & $\begin{array}{l}\text { Residents } \\
\text { interview }\end{array}$ & $\begin{array}{l}\text { Single storey building ( } N=319) \text { Double storey } \\
\text { building ( } N=147) \text { Three storey building }(N=43) \text { Four } \\
\text { storey building }(N=13) \text { Five storey building }(N=01)\end{array}$ \\
\hline$x_{5}$ & $\begin{array}{l}\text { At which } \\
\text { floor do you } \\
\text { live }\end{array}$ & $\begin{array}{l}\text { Jones, Fuertes, \& } \\
\text { Lomas, } 2015\end{array}$ & $\begin{array}{l}\text { Residents } \\
\text { interview }\end{array}$ & $\begin{array}{l}\text { Ground floor }(N=63) \text { First floor }(N=27) \text { Second floor } \\
(N=24) \text { Third floor }(N=3) \text { Multiple floors }(N=3) \text { All } \\
\text { floors }(N=403)\end{array}$ \\
\hline$x_{6}$ & Building age & $\begin{array}{l}\text { Jones, Fuertes, \& } \\
\text { Lomas, 2015; } \\
\text { Huebner, Shipworth, } \\
\text { Hamilton, Chalabi \& } \\
\text { Oreszczyn, } 2016\end{array}$ & $\begin{array}{l}\text { Residents } \\
\text { interview }\end{array}$ & $M=14.94, S D=8.82$ \\
\hline$x_{7}$ & $\begin{array}{l}\text { Type of } \\
\text { ceiling }\end{array}$ & $\begin{array}{l}\text { Huebner, Shipworth, } \\
\text { Hamilton, Chalabi \& }\end{array}$ & $\begin{array}{l}\text { Residents } \\
\text { interview }\end{array}$ & Standard $(N=517)$, False $(N=6)$ \\
\hline$x_{8}$ & $\begin{array}{l}\text { Material } \\
\text { used for } \\
\text { walls }\end{array}$ & Oreszczyn, 2016 & & $\begin{array}{l}\text { Bricks }(\mathrm{N}=338) \text {, PCC Concrete blocks without steel } \\
\qquad(\mathrm{N}: 185)\end{array}$ \\
\hline$x_{9}$ & $\begin{array}{l}\text { Material } \\
\text { used for roof }\end{array}$ & Primary Surveys & $\begin{array}{l}\text { Residents } \\
\text { interview }\end{array}$ & Lanter $(N=520)$, Corrugated Metal sheets $(N=3)$ \\
\hline$x_{10}$ & $\begin{array}{l}\text { Sides of } \\
\text { home } \\
\text { directly } \\
\text { exposed to } \\
\text { sun }\end{array}$ & $\begin{array}{c}\text { Abanda \& Bayers, } \\
2016\end{array}$ & Site Visit & $M=3.30, S D=0.84$ \\
\hline$x_{11}$ & $\begin{array}{l}\text { No. of } \\
\text { windows in } \\
\text { each room }\end{array}$ & $\begin{array}{c}\text { Kavousian, Rajagopal, } \\
\text { \& Fischer, 2013; } \\
\text { Huebner, Shipworth, } \\
\text { Hamilton, Chalabi \& } \\
\text { Oreszczyn, } 2016\end{array}$ & $\begin{array}{l}\text { Residents } \\
\text { interview }\end{array}$ & $M=2.66, S D=0.87$ \\
\hline$x_{12}$ & $\begin{array}{l}\text { Living in this } \\
\text { house since }\end{array}$ & Primary Surveys & $\begin{array}{l}\text { Residents } \\
\text { interview }\end{array}$ & $M=18.65, S D=88.59$ \\
\hline$x_{13}$ & $\begin{array}{l}\text { Building } \\
\text { Ownership }\end{array}$ & $\begin{array}{l}\text { Huebner, Shipworth, } \\
\text { Hamilton, Chalabi \& } \\
\text { Oreszczyn, } 2016\end{array}$ & $\begin{array}{l}\text { Residents } \\
\text { interview }\end{array}$ & Own $(N=373)$, Rented $(N=150)$ \\
\hline$x_{14}$ & $\begin{array}{l}\text { Total floor } \\
\text { area }\end{array}$ & $\begin{array}{l}\text { Baker \& Rylatt, 2008; } \\
\text { William \& Gomez, } \\
\text { 2016; Huebner, } \\
\text { Shipworth, Hamilton, } \\
\text { Chalabi \& Oreszczyn, } \\
2016\end{array}$ & $\begin{array}{l}\text { Residents } \\
\text { interview }\end{array}$ & $M=96.97, S D=22.13$ \\
\hline
\end{tabular}

Table 3. Demographic variables.

\begin{tabular}{|c|c|c|c|c|}
\hline Variable & Variable description & Reference & Data source & $\begin{array}{c}\text { Categories } \\
\mathrm{N} \text { (Frequency) }\end{array}$ \\
\hline$x_{15}$ & $\begin{array}{c}\text { No. of residents above } \\
15 \text { years }\end{array}$ & $\begin{array}{c}\text { Tso \& Yao, 2003; } \\
\text { Jones, Fuertes, \& } \\
\text { Lomas, } 2015\end{array}$ & Resident's interview & $\begin{array}{c}1(0), 2(26), 3 \\
(122), 4(150), 5 \\
(124), 6(57), 7 \\
(30), 8(10), 9(03)\end{array}$ \\
\hline$x_{16}$ & $\begin{array}{l}\text { No. of residents } \\
\text { below15 years }\end{array}$ & $\begin{array}{c}\text { Tso \& Yao, 2003; } \\
\text { Jones, Fuertes, \& } \\
\text { Lomas, } 2015\end{array}$ & Resident's interview & $\begin{array}{c}1(80), 2(53), 3 \\
(86), 4(87), 5(39), \\
6(15), 7(05), 8 \\
(0), 9(0)\end{array}$ \\
\hline$x_{17}$ & $\begin{array}{l}\text { No. of working } \\
\text { residents }\end{array}$ & Primary survey & Resident's interview & $\begin{array}{c}1(115), 2(235), 3 \\
(124), 4(37), 5 \\
(08),\end{array}$ \\
\hline
\end{tabular}


Table 4. Socio-economic Variables.

\begin{tabular}{|c|c|c|c|c|}
\hline $\begin{array}{c}\text { Variable } \\
\text { Abbreviation }\end{array}$ & Variable & Reference & Data source & $\begin{array}{c}\text { Categories } \\
\mathrm{N} \text { (Frequency) }\end{array}$ \\
\hline$x_{18}$ & $\begin{array}{l}\text { How would you grade } \\
\text { your family socially }\end{array}$ & Primary Survey & Resident's interview & $\begin{array}{c}\text { Casually social (372), } \\
\text { Highly social (151) }\end{array}$ \\
\hline$x_{19}$ & $\begin{array}{c}\text { Total family } \\
\text { income/month }\end{array}$ & $\begin{array}{c}\text { Jones, Fuertes, \& } \\
\text { Lomas, 2015; } \\
\text { Shabunko, Lim, } \\
\text { Brahim \& Mathew, } \\
2014\end{array}$ & Resident's interview & $\begin{array}{c}0-10 \mathrm{k}(2), 11 \mathrm{k}-50 \mathrm{k} \\
(13), 50 \mathrm{k}-100 \mathrm{k}(110), \\
101 \mathrm{k}-150 \mathrm{k}(27), \text { above } \\
150 \mathrm{k}(16), \text { No } \\
\text { response (355) }\end{array}$ \\
\hline$x_{20}$ & $\begin{array}{c}\text { Are you a local resident } \\
\text { of the area? }\end{array}$ & Primary Survey & Resident's interview & Yes (416), No (107) \\
\hline$x_{21}$ & $\begin{array}{l}\text { Average no. of week } \\
\text { family remain away }\end{array}$ & Primary Survey & Resident's interview & $\begin{array}{l}0(416), 1-4(78), 5-8 \\
(17), 9-12(12)\end{array}$ \\
\hline$x_{22}$ & No. of cars/vehicles & $\begin{array}{l}\text { Ghedamsi, R., } \\
\text { Settou, N., Gouareh, } \\
\text { A., Khamouli, A., } \\
\text { Saifi, N., Recioui, B., } \\
\text { \& Dokkar, } 2016\end{array}$ & Resident's interview & $\begin{array}{c}0(91), 1(287), 2(127), \\
3(16), 4(2),\end{array}$ \\
\hline$x_{23}$ & No. of full time servants & $\begin{array}{l}\text { Ghedamsi, R., } \\
\text { Settou, N., Gouareh, } \\
\text { A., Khamouli, A., } \\
\text { Saifi, N., Recioui, B., } \\
\text { \& Dokkar, } 2016\end{array}$ & Resident's interview & $\begin{array}{c}0(473), 1(48), 2(1), 3 \\
(0), 4(1),\end{array}$ \\
\hline$x_{24}$ & $\begin{array}{c}\text { No of hours part time } \\
\text { servant works at your } \\
\text { home }\end{array}$ & Primary Survey & Resident's interview & $\begin{array}{c}0(242), 1(07), 2(63) \\
3(93), 4(64), 5(35), 6 \\
(14), 7(05)\end{array}$ \\
\hline & & & & $\begin{array}{c}\text { Categories } \\
\text { Frequency }(\mathrm{N}), \\
\text { Mean }(M) \\
\text { Standard }\end{array}$ \\
\hline Variable & Variable description & Reference & Data source & Deviation (SD) \\
\hline$x_{25}$ & $\begin{array}{l}\text { Total number of ceiling } \\
\text { fans }\end{array}$ & Nation Master, 2018 & Resident's interview & $\begin{array}{c}1(0), 2(02), 3 \\
(28), 4(70), 5 \\
(128), 6(120), 7 \\
(91), 8(44), 9 \\
(24), 10(09), 11 \\
(05), 12(02)\end{array}$ \\
\hline$x_{26}$ & $\begin{array}{l}\text { Number of pedestal } \\
\text { fans }\end{array}$ & Nation Master, 2018 & Resident's interview & $\begin{array}{c}0(364), 1(122), 2 \\
(30), 3(5), 6(2)\end{array}$ \\
\hline$x_{27}$ & $\begin{array}{l}\text { Average no. of lights in } \\
\text { each room }\end{array}$ & $\begin{array}{l}\text { Nation Master, 2018; } \\
\text { Jones, Fuertes, \& } \\
\text { Lomas, } 2015\end{array}$ & Resident's interview & $\begin{array}{c}1(26), 2(398), 3 \\
\text { (89) }\end{array}$ \\
\hline$x_{28}$ & Type of lights installed & $\begin{array}{l}\text { Jones, Fuertes, \& } \\
\text { Lomas, } 2015\end{array}$ & Resident's interview & $\begin{array}{c}\text { Mixed (424), } \\
\text { Energy Saver (85), } \\
\text { Tube light (14), } \\
\text { LEDs (0) }\end{array}$ \\
\hline$x_{29}$ & No. of AC installed & Tso \& Yao, 2003; & Resident's interview & $\begin{array}{c}0 \text { (332), } 1(106), 2 \\
(69), 3(16)\end{array}$ \\
\hline$x_{30}$ & No. of Electric geysers & Tso \& Yao, 2003 & Resident's interview & $\begin{array}{c}0 \text { (179), } 1 \text { (245), } 2 \\
(95), 3(04)\end{array}$ \\
\hline$x_{31}$ & No. of room coolers & & Resident's interview & $\begin{array}{c}0(241), 1(181), 2 \\
(98), 3(03)\end{array}$ \\
\hline$x_{32}$ & No. of Fridges & $\begin{array}{l}\text { HDIP, 2015; Jones, } \\
\text { Fuertes, \& Lomas, } \\
2015\end{array}$ & Resident's interview & $\begin{array}{c}0(08), 1(477), 2 \\
(44), 3(01)\end{array}$ \\
\hline$x_{33}$ & No. of electric heaters & HDIP, 2015 & Resident's interview & $\begin{array}{c}0 \text { (298), } 1(173), 2 \\
(51), 3(01)\end{array}$ \\
\hline$x_{34}$ & No. of TVs & HDIP, 2015 & Resident's interview & $\begin{array}{c}0(04), 1(218), 2 \\
(257), 3(37), 4 \\
(07)\end{array}$ \\
\hline
\end{tabular}




\begin{tabular}{|c|c|c|c|c|}
\hline$x_{35}$ & No. of computers & HDIP, 2015 & Resident's interview & $\begin{array}{c}0(80), 1(242), 2 \\
(158), 3(33), 4 \\
(09), 5(01)\end{array}$ \\
\hline$x_{36}$ & No. of Electric Cookers & $\begin{array}{c}\text { Nation Master; Tso \& } \\
\text { Yao, } 2003\end{array}$ & Resident's interview & $\begin{array}{c}0(372), 1(150), 2 \\
(01)\end{array}$ \\
\hline$x_{37}$ & Do you have UPS & Primary survey & Resident's interview & $\begin{array}{l}\text { Yes (259), No } \\
(264)\end{array}$ \\
\hline$x_{38}$ & $\begin{array}{l}\text { Size of Uninterrupted } \\
\text { Power Supply }\end{array}$ & Primary survey & Resident's interview & $\begin{array}{l}M=540.73 \\
S D=587.81\end{array}$ \\
\hline$x_{39}$ & $\begin{array}{l}\text { No of Washing } \\
\text { machines }\end{array}$ & $\begin{array}{l}\text { Tso \& Yao, 2003; } \\
\text { Jones, Fuertes, \& } \\
\text { Lomas, } 2015\end{array}$ & Resident's interview & $\begin{array}{c}0(06), 1(506), 2 \\
(11)\end{array}$ \\
\hline$x_{40}$ & $\begin{array}{l}\text { Number of washing } \\
\text { machine dryer }\end{array}$ & $\begin{array}{c}\text { Nation Master; Tso \& } \\
\text { Yao, } 2003\end{array}$ & Resident's interview & $\begin{array}{c}0(166), 1(354), 2 \\
(01), 4(02)\end{array}$ \\
\hline$x_{41}$ & No of water dispenser & Primary survey & Resident's interview & 0 (422), 1 (101), \\
\hline$x_{42}$ & $\begin{array}{c}\text { Number of vacuum } \\
\text { cleaner }\end{array}$ & Tso \& Yao, 2003 & Resident's interview & $\begin{array}{c}0(324), 1(198), 2 \\
(01)\end{array}$ \\
\hline$x_{43}$ & $\begin{array}{l}\text { Number of water } \\
\text { Pump Motors }\end{array}$ & Tso \& Yao, 2003 & Resident's interview & 0 (177), 1 (346) \\
\hline$x_{44}$ & No of electric Irons & Primary survey & Resident's interview & $\begin{array}{c}0(02), 1(283), 2 \\
(232), 3(06)\end{array}$ \\
\hline$x_{45}$ & $\begin{array}{c}\text { No. of Microwave } \\
\text { Oven }\end{array}$ & $\begin{array}{l}\text { Jones, Fuertes, \& } \\
\text { Lomas, } 2015\end{array}$ & Resident's interview & $\begin{array}{c}0(207), 1(299), 2 \\
(15), 3(02)\end{array}$ \\
\hline$x_{46}$ & Generator & Primary survey & Resident's interview & $\begin{array}{l}M=28.11 \\
S D=204.11\end{array}$ \\
\hline$x_{50}$ & $\begin{array}{c}\text { Do you have Solar } \\
\text { Panels installed at your } \\
\text { home }\end{array}$ & Primary survey & Resident's interview & Yes (29), No (494) \\
\hline$x_{51}$ & $\begin{array}{c}\text { Do you have solar } \\
\text { geysers installed at } \\
\text { your home }\end{array}$ & Primary survey & Resident's interview & Yes (11), No (512) \\
\hline$x_{52}$ & $\begin{array}{l}\text { Is electricity meter } \\
\text { shared? }\end{array}$ & Primary survey & Resident's interview & Yes (0), No (523) \\
\hline
\end{tabular}

\section{Dependent variable}

The annual electricity consumption in kilowatt hours $(\mathrm{kWh})$ is the dependent variable, the data of the dependent variable were collected from the residents in the form of electricity bills. The data having the constant electricity consumption throughout the year were excluded. The mean of the annual electricity consumption is $2468.88 \mathrm{kWh}$ and the standard deviation is 8.25 . The trend of average monthly electricity consumption in all 523 throughout the year is shown in Figure 4.

Figure 4. Average Monthly Electricity Consumption Profile of a domestic building in Pakistan

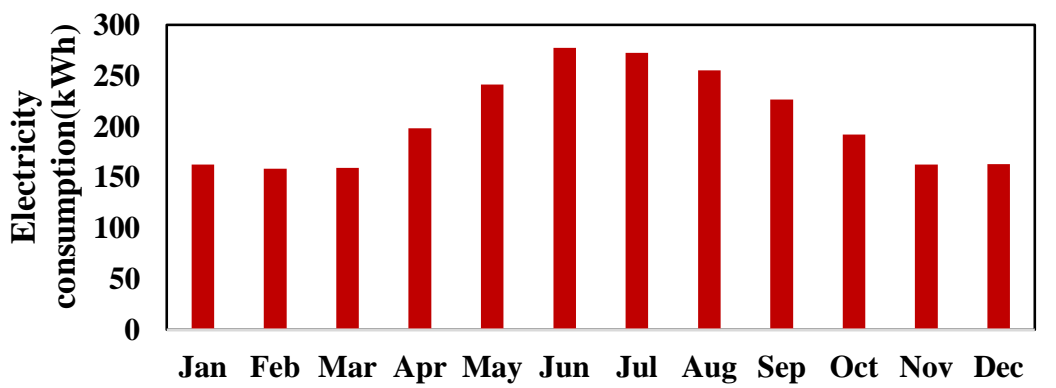

Months

The trend clearly shows high electricity consumption during summer months. This is mainly because of cooling load. In winter, electricity consumption trend remains consistent. 


\section{Multiple regression analysis}

When multiple predictors are used to predict the dependent variable's value, multiple regression is employed. A number of researchers have employed MRA in their studies to establish relationship among dependent and independent variables. While performing MR analysis, it is important to check whether there are any two or more than two independent variables collinear to each other. One method of checking co-linearity among variables is the Pearson Correlation Coefficient and is usually denoted by $\mathrm{R}$. Two variables are strongly collinear if $\mathrm{R}=>0.6$ and vice versa. Another check that helps in identifying the significant variables is t-stat check. In MR analysis, variables having t-stat values $\Rightarrow 1.96$ or $p$-values $<=0.05$ are considered significant and vice versa. SPSS software provides a detailed analysis showing $\mathrm{R}, \mathrm{t}$-stat and $\mathrm{p}$-values.

\section{Results}

To observe the effect of independent variables on annual electricity consumption (Y) MR analysis was performed using SPSS software. Model fitness, $\mathrm{R}^{2}$ change, Pearson correlation and confidence interval $95 \%$ were observed. The colinearity was observed between independent variables using Pearson correlation. Sixteen independent variables were found collinear, and some of these were dropped after observing their low t-state value. 17 variables were found significant and strong predictors. These variables are and their significance is demonstrated through t-stat values and p-values as shown in Table 6.

\begin{tabular}{|c|c|c|c|c|}
\hline Description of Variable & $\begin{array}{c}\text { Variable } \\
\text { (Constant) }\end{array}$ & $\begin{array}{c}\text { B } \\
-295.308 \\
\end{array}$ & $\begin{array}{c}\text { t-stat } \\
\text { value } \\
-1.713 \\
\end{array}$ & $\begin{array}{c}\text { Significance } \\
0.87 \\
\end{array}$ \\
\hline No. of windows in each room & $\mathrm{X}_{11}$ & 81.657 & 2.971 & .003 \\
\hline $\begin{array}{l}\text { No. of residents above } 15 \\
\text { years }\end{array}$ & $\mathrm{x}_{15}$ & 61.743 & 3.304 & .001 \\
\hline No. of cars/vehicles & $x_{22}$ & 81.821 & 2.222 & .027 \\
\hline Total number of ceiling fans & $\mathbf{x}_{25}$ & 87.004 & 5.016 & .000 \\
\hline Number of pedestal fans & $x_{26}$ & 175.236 & 5.063 & .000 \\
\hline Number of lights in each room & $x_{27}$ & 182.215 & 8.313 & .000 \\
\hline No. of AC installed & $\mathbf{X}_{29}$ & 347.336 & 10.551 & .000 \\
\hline No. of Electric geysers & $\mathrm{x}_{30}$ & 87.343 & 2.226 & .026 \\
\hline No. Of electric heaters & $x_{33}$ & 76.971 & 1.959 & .051 \\
\hline No. Of TVs & $x_{34}$ & 160.926 & 4.309 & .000 \\
\hline No. of computers & $X_{35}$ & 125.343 & 4.334 & .000 \\
\hline No. of Electric Cookers & $x_{36}$ & -197.615 & -3.350 & .001 \\
\hline No of Washing machines & $\mathbf{X}_{39}$ & 283.140 & 2.147 & .032 \\
\hline No of water dispenser & $\mathrm{X}_{41}$ & 215.825 & 3.477 & .001 \\
\hline Number of vacuum cleaner & $\mathrm{X}_{42}$ & 166.044 & 3.087 & .002 \\
\hline No. of Microwave Oven & $\mathrm{X}_{45}$ & 184.216 & 3.863 & .000 \\
\hline Solar Panels installed at home & $X_{50}$ & -372.500 & -3.697 & .000 \\
\hline
\end{tabular}

\section{Electricity Usage Intensity (EUI)}

The mean and median values of the EUI $\left(\mathrm{kWh} / \mathrm{m}^{2}\right)$ are shown in Figure 5 whereas the mean and median values of EUI ( $\mathrm{kWh} /$ resident) are presented in Figure 6. Due to the skewed nature of the data distribution, the median values of EUI have been selected for the benchmarking purpose. The median values of EUI are $24 \mathrm{kWh} / \mathrm{m}^{2}$ and $349 \mathrm{kWh} / \mathrm{resident}$ respectively and therefore, these values have been selected as the benchmarks for the electricity consumption in the domestic buildings of Pakistan. The values of EUI $\left(\mathrm{kWh} / \mathrm{m}^{2}\right)$ were compared with same of Bangladesh and India. The EUI of Bangladesh residential sector is $32 \mathrm{kWh} / \mathrm{m}^{2}, 13 \%$ more electricity than EUI of Pakistan (Ahsan, Soebator \& Williamson, 2014) whereas for India it ranges between $12-36 \mathrm{kWh} / \mathrm{m}^{2}$ (GBPN, 2014). 

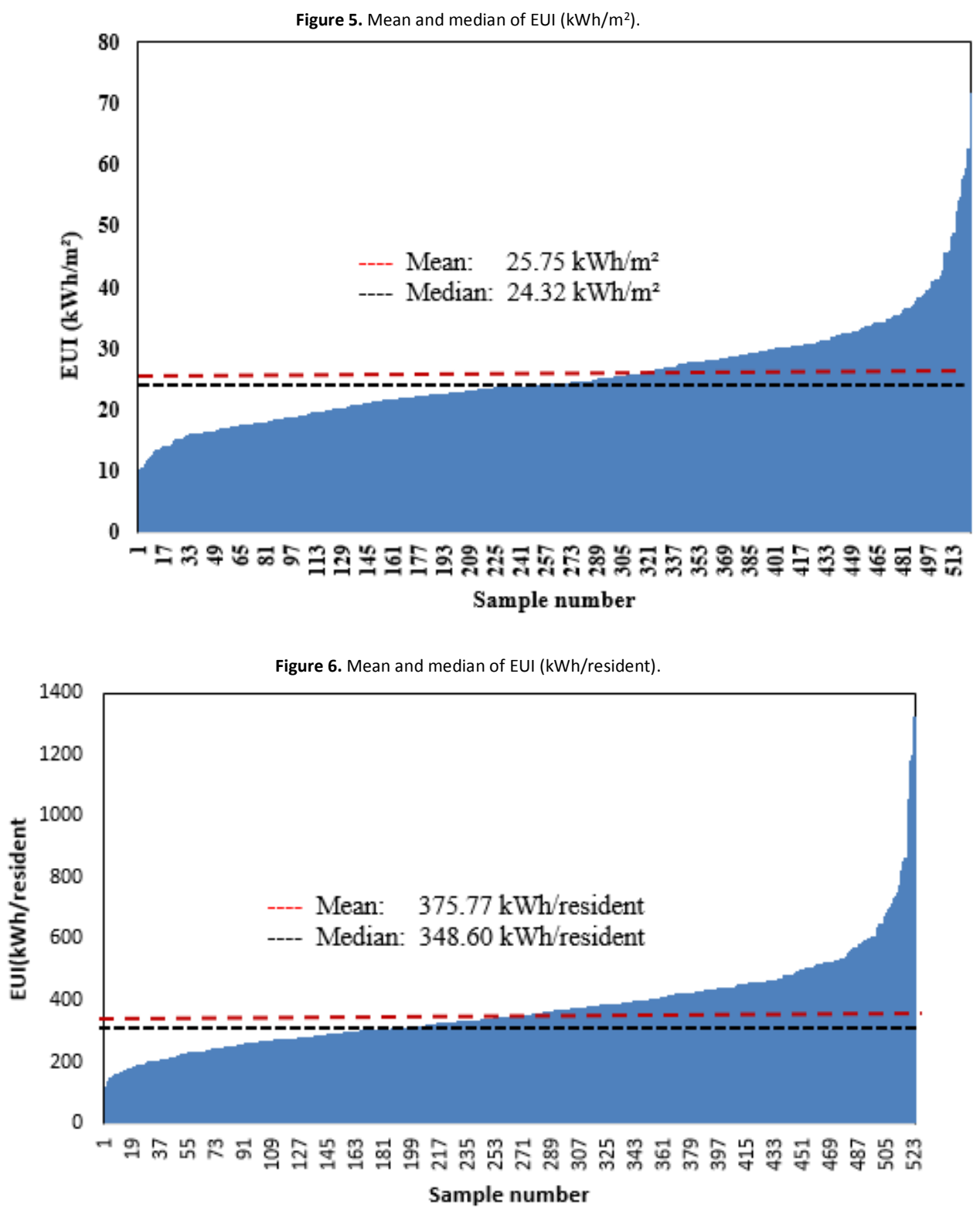

Conclusions

The aim of this study was to observe the impact of dwelling, socio-economic, demographic and appliances variables on annual electricity consumption of residential sector in Pakistan and to establish a EUI value. With the help of multiple regression analysis the impacts of above stated variables were found.

- It has been found that 17 variables have significant impact on annual electricity consumption.

- 15 variables (one from dwelling category, one from socio-economic, one from demographic and 12 variables from appliances category) have significant positive impact on annual electricity consumption. The variables with positive impact are, No. of windows in each room, No. of residents above 15 years, No. of cars/ vehicles, Total number of ceiling fans, Number of pedestal fans, Number of lights in each room, No. of AC installed, No. of Electric geysers, No. of electric heaters, No. of TVs, No. of computers, No of Washing machines, No of water dispensers, Number of vacuum cleaner, and No. of Microwave Ovens.

- Two variables, i.e. No. of Electric Cookers and No. of Solar Panels installed at home have significant negative impact on electricity consumption.

- Further through a univariate analysis, it has been found that annual EUI values are $24 \mathrm{kWh} / \mathrm{m}^{2}$ and $349 \mathrm{kWh} / \mathrm{resident}$. 
Abanda, F. H., \& Byers, L. (2016). An investigation of the impact of building orientation on energy consumption in a domestic building using emerging BIM (Building Information Modelling). Energy, 97, 517-527.

Ahsan T, Soebator V, Williamson T. (2014). Key predictors of annual electricity use in high-rise residential apartments in Dhaka, Bangladesh. Retrieved from http://anzasca.net/wp-content/uploads/2014/12/11_41_18.pdf

Amber, K. P., Aslam, M. W., \& Hussain, S. K. (2015). Electricity consumption forecasting models for administration buildings of the UK higher education sector. Energy and Buildings, 90, 127-136.

Baker, K. J., \& Rylatt, R. M. (2008). Improving the prediction of UK domestic energy-demand using annual consumption-data. Applied Energy, 85(6), 475-482.

Domínguez, M., Alonso, S., Morán, A., Prada, M. A., \& Fuertes, J. J. (2015). Dimensionality reduction techniques to analyze heating systems in buildings. Information Sciences, 294, 553-564.

Durkman A, Jackson T. (2008). Household energy consumption in the UK: A highly geographically and socio-economically disaggregated model. Energy Policy 36, 3177- 3192 .

EIA (2015) U.S. Energy Information Administration. Drivers of U.S. Household Energy Consumption1980-2009. Retrieved from https://www.eia.gov/analysis/studies/buildings/households/pdf/drivers hhec.pdf

Enerdata (2018) Electricity residential consumption. Retrieved from https://yearbook,enerdata.net/\#electricity-residential-consumption-data-byregion.html

Government of Pakistan, Ministry of Finance (2006) Pakistan Economic Survey, Energy. Retrieved from http://www.finance.gov.pk/survey/chapters/15-Energy.pdf

GBPN (2014) Residential buildings in India: energy use projections and savings potentials. Retrieved from http://www.gbpn.org/sites/default/files/08.\%20INDIA\%20Baseline_TR_low.pdf

Genjo, K., Tanabe, S. I., Matsumoto, S. I., Hasegawa, K. I., \& Yoshino, H. (2005). Relationship between possession of electric appliances and electricity for lighting and others in Japanese households. Energy and Buildings, 37(3), 259-272.

Ghedamsi, R., Settou, N., Gouareh, A., Khamouli, A., Saifi, N., \& Recioui, B. (2015, March). Estimating the energy consumption in building sector in Algeria using bottom-up mode. In Renewable Energy Congress (IREC), 2015 6th International (pp. 1-6). IEEE.

Ghedamsi, R., Settou, N., Gouareh, A., Khamouli, A., Saifi, N., Recioui, B., \& Dokkar, B. (2016). Modeling and forecasting energy consumption for residential buildings in Algeria using bottom-up approach. Energy and Buildings, 121, 309-317.

HDIP (2015). Hydrocarbon Development Institute of Pakistan, Pakistan Energy Year Book. Retrieved from http://www.kpkep.com/documents/Pakistan\%20Energy\%20Yearbook\%202015.pdf

Huebner, G., Shipworth, D., Hamilton, I., Chalabi, Z., \& Oreszczyn, T. (2016). Understanding electricity consumption: A comparative contribution of building factors, socio-demographics, appliances, behaviours and attitudes. Applied energy, 177, 692-702.

IEA (2008). Worldwide trends in energy use and efficiency. Retrieved from https://www.iea.org/publications/freepublications/publication/Indicators_2008.pdf

Jones, R. V., Fuertes, A., \& Lomas, K. J. (2015). The socio-economic, dwelling and appliance related factors affecting electricity consumption in domestic buildings. Renewable and Sustainable Energy Reviews, 43, 901-917.

Kavousian, A., Rajagopal, R., \& Fischer, M. (2013). Determinants of residential electricity consumption: Using smart meter data to examine the effect of climate, building characteristics, appliance stock, and occupants' behavior. Energy, 55, 184-194.

Leahy E, Lyons S. (2010). Energy use and appliance ownership in Ireland. Energy Policy, 38(8), 4265-79.

Nasir, M., Tariq, M. S., \& Arif, A. (2008). Residential demand for electricity in Pakistan. The Pakistan Development Review, 457-467. Retrieve from http://www.pide.org.pk/psde24/pdf/03.pdf

Nation Master (2018). Electricity consumption per capita: Countries compared. Retrieved from http://www.nationmaster.com/countryinfo/stats/Energy/Electricity/Consumption/Per-capita

Sanquist, T. F., Orr, H., Shui, B., \& Bittner, A. C. (2012). Lifestyle factors in US residential electricity consumption. Energy Policy, 42, 354-364.

Shabunko, V., Lim, C. M., Brahim, S., \& Mathew, S. (2014). Developing building benchmarking for Brunei Darussalam. Energy and Buildings, 85, 79-85. Stern, D. I. (1993). Energy and economic growth in the USA: a multivariate approach. Energy economics, 15(2), 137-150.

Tiwari, P. (2000). Architectural, demographic, and economic causes of electricity consumption in Bombay. Journal of Policy Modeling, 22(1), 81-98.

Tso, G. K., \& Yau, K. K. (2003). A study of domestic energy usage patterns in Hong Kong. Energy, 28(15), 1671-1682. 